DISCUSSION PAPER SERIES

The impact of terrorism on expectations, trust and happiness

The Case of the November 13 attacks in Paris, France

Tom Coupe (Kyiv School of Economics) 
The impact of terrorism on expectations, trust and happiness

The Case of the November 13 attacks in Paris, France

\title{
Tom Coupe
}

\begin{abstract}
We use quasi-experimental evidence to measure the impact of the November 13, 2015 attacks in Paris, France on various channels through which terrorism can affect the economy. The evidence suggest the attacks reduced optimism and increased trust in the national government but did not affect current life satisfaction nor political orientation.
\end{abstract}




\section{$\underline{\text { Introduction }}$}

There is by now a sizeable literature that studies the economic impact of terrorism. For example, Abadie and Gardeazabal (2003) show that terrorism cost the Basque Country 10\% of its per-capita GDP. Tavares (2004), using a large panel of countries, however, finds the output cost of terrorist attacks to be small, and smaller for more democratic countries.

Terrorist acts typically only directly affect a small number of individuals and organizations, so to have a sizeable influence on the overall economy, they would need to have a psychological impact on the wider population or affect political outcomes (Krueger (2008, p.8 -9)).

Examples of the impact of terrorism on political outcomes are Montalvo (2011), who finds a significant effect of the 2004 Madrid Bombings on the outcome of the Spanish elections, and Bozzoli and Mueller (2009), who find that the July 2005 London Bombings increased people's willingness to trade off liberties for security.

There are several examples of the impact of terrorism on the mood of the population. Metcalfe et al. (2011) find a significant negative effect of the 9/11 attack on the World Trade center on happiness in the UK, while Clark and Stancanelli (2016) find a significant negative effect of the Boston Marathon Bombing on happiness in the US. Romanov et al (2012), however, find little evidence of terrorism on life satisfaction in Israel. These studies capture the impact of terrorism by comparing answers of respondents interviewed just before the terrorist attacks to the answers of respondents interviewed just after the attacks.

Such quasi-experimental methodological approach is also used to assess the impact of terrorism on trust. Dinesen and Jaeger (2013), for example, find that the 2004 Madrid Bombings had a shortlived positive effect on trust in political institutions. Wollebæk et al. ( 2012) similarly document an increase in interpersonal and institutional trust after the Utoya murders in Norway while Gates and Justesen (2016) find a decrease in trust in the president after a Tuareg attack in Mali.

In this note, we estimate the impact of the November 13, 2015 attacks in Paris, France. On November 13, terrorists attacked 4 different places in Paris, killing 130 people and injuring over 350 people. While France had suffered an earlier attack on Charlie Hebdo, in January 2015, the November 2015 attack was the first Islamist attack in France aimed not at a selected group of people, but rather aimed to maximize victims in the population.

We analyze the channels that can cause an economic impact by analyzing how the attack influenced the mood, expectations and trust of the French. We use the November 2015 Eurobarometer survey, which interviewed about 1000 French people data in the period November 7-November 16, 2015. About $90 \%$ of the interviews took place on or before November 13, and $10 \%$ after the attack (mainly on Saturday November the $14^{\text {th }}$ ). We also use the Eurobarometer survey done weeks before the attack (October 17-26, 2015) and the survey done weeks after (November $28^{\text {th }}$-December $7^{\text {th }}$, 2015). Using these additional surveys we can assess whether possible immediate effects of the attack persist; though, admittedly, the longer the period between the attack and the survey period, the more likely our outcome variables are not only affected by the attack itself.

All 3 surveys included questions on life satisfaction, on whether the country goes in the right direction and on one's political orientation. The November survey includes additional questions on the importance of terrorism, on quality of life now and in the past, on confidence in the future and on trust in various political institutions.

\section{Econometric Analysis.}

While we have data from different periods in time, the surveys do not follow the same people over time. Hence, changes over time could be due to changes in the sample composition. We therefor 
report the results of a regression analysis which control for differences in the sample by including a fixed set of control variables:

- Age

- A dummy for women

- A dummy for rural areas

- A set of dummies reflecting different levels of education

- A dummy for respondents who regularly have difficulties paying bills

- A set of dummies reflecting the relationship status

- A set of dummies reflecting the employment status

These variables are typical control variables in studies of happiness, expectations, trust and political views. For example, Jacobsen et al. (2014) study the relationship between optimism and gender, Powdthavee et al. (2015) between happiness and education, Stevenson and Wolfers (2008) between happiness and the economic situation, Sutter and Kocher (2007) between age and trust, Yang and Zeng (2016) between the urban/rural divide and trust.

\section{Analysis.}

Column Ia and Ib of table 1 give the results of an ordered probit model analyzing how satisfied a respondent is with the life (s)he leads. Respondents had a choice between four possible answers, ranging from 'Very Satisfied' to Not At All Satisfied. Table 1 gives the average marginal effects on the probability of answering 'Very satisfied (Ia)'or 'Fairly satisfied' (Ib). Given marginal effects need to sum to one, one can also infer from the table the marginal effects on the probability of being in the remaining categories. The table shows that the probability of a respondent answering (s)he is very satisfied, is increasing over time, with no signs of the attack changing this trend. The same conclusion follows from analyzing the question whether a respondent thinks, currently, the country is going in the right direction: compared to weeks before the attack, more people think the country is going in the right direction but this trend already started before the attack (column IIa and IIb).

Similarly, we do not find that the attacks affect the probability people were happy with the quality of life (columns IIIa and IIIb) nor that it made respondents more likely to agree with the statement that qualify of life before was better (columns IVa and IVb).

We do find, however, that the attacks negatively affected expectations: we find that, after the attack, the probability respondents expect the quality of life to become better in the next 12 months, is 7 percentage points lower than before (column IVa). Compared to the $18 \%$ of respondents who believed the quality of life to become better before the attack, this $7 \%$ points change is sizeable. Similarly, we find that respondents are 6 percentage points less likely to 'totally agree' with the statement they are confident in the future and 6 percentage points less likely to 'tend to agree' with such statement. This decrease of 12 percentage points in the probability of agreeing that one is confident in the future is sizeable when compared to the $53 \%$ of respondents agreeing with this statement before the attack.

Focusing on the possible political impact of the attacks, we find that the chance a respondent indicates terrorism is among the two most important problems the country faces, increases by 29.4 percentage points (column VII), while the chance a respondent indicates terrorism is among the two most important problems (s)he personally faces increases by 6.1 percentage points (column VIII). Compared to initial levels of $15 \%$ and $3.5 \%$, these are massive increases. Finally, we find that the attacks significantly and positively affected trust in the national government, with the increase being a sizeable 13 percentage point (column XI), which is almost a doubling compared to the base level of $18 \%$. We do not find a significant impact on individuals' political left/right orientation (column $\mathrm{XII}$ ), nor on trust in political parties (column IX) or local governments (column X). 


\section{Conclusion}

The quasi-experimental evidence presented here suggests the November 13, 2015 terrorist attacks in Paris, France, worsened expectations about the future, increased trust in the national government and brought terrorism up in people's agenda. But they did not affect current satisfaction with life nor with the direction in which the country is going, nor the political orientation of respondents.

\section{References}

Abadie Alberto and Javier Gardeazabal. 2003. "The Economic Costs of Conflict: A Case Study of the Basque Country” The American Economic Review, 93(1), p. 113-132.

Bozzoli, Carlos and Müller, Cathérine. 2011. "Perceptions and attitudes following a terrorist shock: Evidence from the UK”, European Journal of Political Economy, 27(S1), p. S89-S106.

Clark, Andrew and Elena Stancanelli. 2016. "Individual Well-Being and the Allocation of Time Before and After the Boston Marathon Terrorist Bombing”. PSE Working Paper.

Dinesen, Peter Thisted and Mads Meier Jæger. 2013. "The Effect of Terror on Institutional Trust: New Evidence from the 3/11 Madrid Terrorist Attack.", Political Psychology, 34(6), p. 917-926.

Gates, Scott and Mogens K. Justesen (2016), Political Trust, Shocks, and Accountability: QuasiExperimental Evidence from a Rebel Attack, working paper.

Jacobsen, Ben, John B. Lee, , Wessel Marquering, , Cherry Y. Zhang (2014), 'Gender differences in optimism and asset allocation', Journal of Economic Behavior \& Organization, 107(B), P. 630651.

Krueger, Alan B. 2008. 'What Makes a Terrorist: Economics and the Roots of Terrorism', Princeton University Press.

Metcalfe, Robert, Nattavudh Powdthavee and Paul Dolan (2011), "Destruction and Distress: Using a Quasi-Experiment to Show the Effects of the September 11 Attacks on Mental Well-Being in the United Kingdom”, 121(550), p. F81-F103

Montalvo, Jose. 2011. "Voting after the Bombings: A Natural Experiment on the Effect of Terrorist Attacks on Democratic Elections", The Review of Economics and Statistics, 93(4), p. 1146-1154.

Powdthavee, Nattavudh (Nick), Warn N. Lekfuangfu and Mark Wooden. 2015. 'What's the good of education on our overall quality of life? A simultaneous equation model of education and life satisfaction for Australia', Journal of Behavioural and Experimental Economics, 54, p. 10- 21.

Romanov, Dmitri, AsafZussman, and Noam Zussman, 'Does Terrorism Demoralize? Evidence from Israel', Economica, 79(313), p. 183-198.

Stevenson, Betsey \& Justin Wolfers, 2008. "Economic Growth and Subjective Well-Being: Reassessing the Easterlin Paradox," Brookings Papers on Economic Activity, vol. 39(1), p. 1-102.

Sutter, Matthias and Martin Kocher. 2007. 'Trust and trustworthiness across different age groups', Games and Economic Behavior, 59(2), p. 364-382.

Tavares, Jose. 2004. "The open society assesses its enemies: shocks, disasters and terrorist attacks , Journal of Monetary Economics, 51(5), p. 1039-1070.

Wollebæk, Dag, Bernard Enjolras, Kari Steen-Johnsen, Guro Ødegård. 2012. 'After Utøya: How a High-Trust Society Reacts to Terror-Trust and Civic Engagement in the Aftermath of July 22', PS: Political Science \& Politics, 45(1), p. 32-37. 
Yang, Guanqiong and Shuxian Zeng. 2016. 'The Comparison of Trust Structure between Urban and Rural Residents in China', American Journal of Industrial and Business Management, 6, p. 665673. 


\begin{tabular}{|c|c|c|c|c|c|c|c|c|c|c|c|c|}
\hline \multirow[t]{2}{*}{ Table 1a } & \multicolumn{2}{|c|}{$\begin{array}{l}\text { Current Life } \\
\text { Satisfaction }\end{array}$} & \multicolumn{2}{|c|}{$\begin{array}{l}\text { Currently going in which } \\
\text { direction? }\end{array}$} & \multicolumn{2}{|c|}{ Current Quality of Life } & \multicolumn{2}{|c|}{$\begin{array}{l}\text { Quality of Life was } \\
\text { Better Before }\end{array}$} & \multicolumn{2}{|c|}{$\begin{array}{l}\text { Quality of Life in } \\
\text { the next } 12 \text { months } \\
\text { will be }\end{array}$} & \multicolumn{2}{|c|}{$\begin{array}{c}\text { Do you agree with the } \\
\text { statement that you } \\
\text { have confidence in } \\
\text { future }\end{array}$} \\
\hline & $\begin{array}{c}\text { (Ia) } \\
\text { Very } \\
\text { Satisfied }\end{array}$ & $\begin{array}{c}\text { (Ib) } \\
\text { Fairly } \\
\text { Satisfied }\end{array}$ & $\begin{array}{l}\text { (IIa) } \\
\text { Right }\end{array}$ & $\begin{array}{l}\text { (IIb) } \\
\text { Neither } \\
\text { Right nor } \\
\text { Wrong }\end{array}$ & $\begin{array}{c}\text { (IIIa) } \\
\text { Very Good }\end{array}$ & $\begin{array}{l}\text { (IIIb) } \\
\text { Fairly Good }\end{array}$ & $\begin{array}{l}(\text { IVa }) \\
\text { Totally } \\
\text { Agree }\end{array}$ & $\begin{array}{l}\text { (IVb) } \\
\text { Tend to } \\
\text { Agree }\end{array}$ & $\begin{array}{l}\text { (Va) } \\
\text { better }\end{array}$ & $\begin{array}{l}(\mathrm{Vb}) \\
\text { same }\end{array}$ & $\begin{array}{l}\text { (VIa) } \\
\text { Totally } \\
\text { agree }\end{array}$ & $\begin{array}{l}\text { (VIb) } \\
\text { Tend to } \\
\text { agree }\end{array}$ \\
\hline $\begin{array}{l}\text { Immediately } \\
\text { Before the } \\
\text { attack }\end{array}$ & 0.012 & -0.002 & $0.068 * * *$ & $0.043 * * *$ & & & & & & & & \\
\hline & 0.01 & 0 & 0.01 & 0.01 & & & & & & & & \\
\hline $\begin{array}{c}\text { Immediately } \\
\text { After the } \\
\text { attack }\end{array}$ & 0.021 & -0.004 & $0.077 * *$ & $0.048 * * *$ & -0.005 & -0.007 & 0.025 & 0 & $-0.070 * *$ & -0.01 & $-0.058 * *$ & ${ }^{-}-0.060^{* * *}$ \\
\hline & 0.03 & 0.01 & 0.03 & 0.02 & 0.02 & 0.03 & 0.04 & 0 & 0.03 & 0.01 & 0.02 & 0.02 \\
\hline $\begin{array}{c}\text { Weeks After } \\
\text { the attack }\end{array}$ & $0.032 * *$ & $-0.007 * *$ & $0.068 * * *$ & $0.043 * * *$ & & & & & & & & \\
\hline & 0.01 & 0 & 0.01 & 0.01 & & & & & & & & \\
\hline $\mathrm{N}$ & 3015 & 3015 & 2845 & 2845 & 932 & 932 & 932 & 932 & 934 & 934 & 934 & 934 \\
\hline
\end{tabular}

\begin{tabular}{|c|c|c|c|c|c|c|}
\hline Table 1b & Priority for France & Priority for oneself & Political Parties & Local Gov. & National Gov. & Political Orientation \\
\hline & (VII) & (VIII) & $\begin{array}{c}\text { (IX) } \\
\text { Tend to Trust } \\
\end{array}$ & $\begin{array}{c}(\mathrm{X}) \\
\text { Tend to Trust }\end{array}$ & $\begin{array}{c}\text { (XI) } \\
\text { Tend to Trust } \\
\end{array}$ & $\begin{array}{c}\text { XII } \\
\text { Left(1)-Right(10) } \\
\end{array}$ \\
\hline \multirow[t]{2}{*}{$\begin{array}{c}\text { Immediately Before the } \\
\text { attack }\end{array}$} & & & & & & $-0.191 *$ \\
\hline & & & & & & 0.11 \\
\hline \multirow[t]{2}{*}{$\begin{array}{c}\text { Immediately After the } \\
\text { attack }\end{array}$} & $0.294 * * *$ & $0.061 * * *$ & 0.041 & 0.043 & $0.133 * * *$ & -0.145 \\
\hline & 0.03 & 0.02 & 0.03 & 0.06 & 0.04 & 0.27 \\
\hline \multirow[t]{2}{*}{ Weeks After the attack } & & & & & & -0.041 \\
\hline & & & & & & 0.11 \\
\hline $\mathrm{N}$ & 1000 & 1000 & 855 & 888 & 888 & 2358 \\
\hline
\end{tabular}

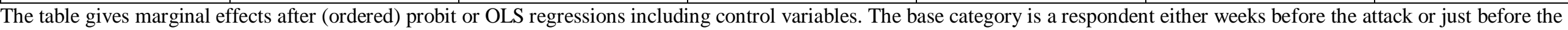
attack depending on data availability. One * indicates significant at the $10 \%, * * 5 \%$ and $* * * 1 \%$ significance level. Full tables can be found in an online appendix. 
Online Appendix

\section{Descriptive Stats Analysis.}

1. On the whole, are you very satisfied, fairly satisfied, not very satisfied or not at all satisfied with the life you lead?

\begin{tabular}{|l|r|r|r|r|}
\hline & Oct. 17-26 & Nov. 7-Nov. 13 & Nov. 14-Nov. 17 & Nov. 28-Dec. 7 \\
\hline \# observations & 1028 & 929 & 97 & 1038 \\
\hline Very Satisfied & 20.91 & 18.95 & 20.62 & 22.83 \\
\hline Fairly Satisfied & 62.16 & 65.77 & 69.07 & 63.58 \\
\hline Not Very Satisfied & 11.38 & 11.84 & 7.22 & 9.83 \\
\hline Not At All Satisfied & 5.16 & 3.34 & 3.09 & 3.66 \\
\hline DK & 0.39 & 0.11 & 0 & 0.1 \\
\hline
\end{tabular}

2. How do you judge the current situation in terms of life quality in France?

\begin{tabular}{|l|r|r|}
\hline & Nov. 7-Nov. 13 & Nov. 14-Nov. 17th \\
\hline \# observations & 929 & 97 \\
\hline Very Good & 6.78 & 8.25 \\
\hline Rather Good & 62.43 & 59.79 \\
\hline Rather Bad & 23.36 & 26.8 \\
\hline Very Bad & 5.38 & 4.12 \\
\hline DK & 2.05 & 1.03 \\
\hline
\end{tabular}

3. At the present time, would you say that, in general, things are going in the right direction or in the wrong direction, in our country?

\begin{tabular}{|l|r|r|r|r|}
\hline & Oct. 17-26 & Nov. 7-Nov. 13 & Nov. 14-Nov. 17 & Nov. 28-Dec. 7 \\
\hline \# observations & 1028 & 929 & 97 & 1038 \\
\hline Right Direction & 7.49 & 13.56 & 12.37 & 13.39 \\
\hline Wrong Direction & 77.82 & 67.38 & 61.86 & 66.18 \\
\hline Neither (spontaneous) & 8.85 & 13.56 & 18.56 & 13.97 \\
\hline DK & 5.84 & 5.49 & 7.22 & 6.45 \\
\hline
\end{tabular}

4. In general, to what extent you agree that, in terms of quality of your life, things were better before.

\begin{tabular}{|l|r|r|}
\hline & Nov. 7-Nov. 13 & Nov. 14-Nov. 17th \\
\hline \# observations & 929 & 97 \\
\hline Totally Agree & 30.89 & 27.84 \\
\hline Tend to Agree & 32.4 & 38.14 \\
\hline Tend to Disagree & 26.7 & 23.71 \\
\hline Totally disagree & 4.52 & 6.19 \\
\hline DK & 5.49 & 4.12 \\
\hline
\end{tabular}


1. What are your expectations for the quality of life in France over the next 12 months. The next 12 months will be

\begin{tabular}{|l|r|r|}
\hline & Nov. 7-Nov. 13 & Nov. 14-Nov. 17 \\
\hline \# observations & 929 & 97 \\
\hline Better & 17.76 & 13.4 \\
\hline Same & 56.94 & 53.61 \\
\hline Worse & 21.53 & 29.9 \\
\hline Dk & 3.77 & 3.09 \\
\hline
\end{tabular}

2. To what extent do you agree with the statement that you have confidence in the future?

\begin{tabular}{|l|r|r|}
\hline & Nov. 7-Nov. 13 & Nov. 14-Nov. 17 \\
\hline \# observations & 929 & 97 \\
\hline Totally Agree & 11.3 & 12.37 \\
\hline Tend to Agree & 41.55 & 28.87 \\
\hline Tend to Disagree & 30.25 & 38.14 \\
\hline Totally disagree & 12.81 & 16.49 \\
\hline DK & 4.09 & 4.12 \\
\hline
\end{tabular}

1. According to you, which are the two most important problems France faces now?

face la France actuellement ?

\begin{tabular}{|l|r|r|}
\hline & Nov. 7-Nov. 13 & Nov. 14-Nov. 17th \\
\hline \# observations & 929 & 97 \\
\hline Does NOT mention terrorism & 84.82 & 48.45 \\
\hline Mentions Terrorism & 15.18 & 51.55 \\
\hline
\end{tabular}

2. According to you, which are the two most important problems you personally face now?

\begin{tabular}{|l|r|r|}
\hline & Nov. 7-Nov. 13 & Nov. 14-Nov. 17th \\
\hline \# observations & 929 & 97 \\
\hline Does NOT mention terrorism & 96.45 & 87.63 \\
\hline Mentions Terrorism & 3.55 & 12.37 \\
\hline
\end{tabular}

1. To what extent do you trust political parties

\begin{tabular}{|l|r|r|}
\hline & Nov. 7-Nov. 13 & Nov. 14-Nov. 17th \\
\hline \# observations & 929 & 97 \\
\hline Tend to trust & 7 & 11.34 \\
\hline Tend not to trust & 88.91 & 83.51 \\
\hline DK & 4.09 & 5.15 \\
\hline
\end{tabular}

2. To what extent do you trust local government

\begin{tabular}{|l|r|r|}
\hline & Nov. 7-Nov. 13 & Nov. 14-Nov. 17th \\
\hline \# observations & 929 & 97 \\
\hline Tend to trust & 43.70 & 47.42 \\
\hline Tend not to trust & 50.38 & 46.39 \\
\hline
\end{tabular}


3. To what extent do you trust the country's government

\begin{tabular}{|l|r|r|}
\hline & Nov. 7-Nov. 13 & Nov. 14-Nov. 17th \\
\hline \# observations & 929 & 97 \\
\hline Tend to trust & 17.55 & 34.02 \\
\hline Tend not to trust & 77.18 & 63.92 \\
\hline DK & 5.27 & 2.06 \\
\hline
\end{tabular}

Interestingly respondents were more likely to trust these political institutions after the attack then before the attack.

\begin{tabular}{|c|c|c|c|c|}
\hline & Oct. 17-26 & Nov. 7-Nov. 13 & Nov. 14-Nov. 17 & Nov. 28-Dec. 7 \\
\hline \# observations & 1028 & 929 & 97 & 1038 \\
\hline Box 1 - left & 3.89 & 4.84 & 3.09 & 3.47 \\
\hline Box 2 & 3.21 & 4.74 & 7.22 & 3.08 \\
\hline Box 3 & 9.92 & 10.87 & 9.28 & 10.21 \\
\hline Box 4 & 7.39 & 8.72 & 7.22 & 8.57 \\
\hline Box 5 & 23.35 & 25.62 & 21.65 & 25.63 \\
\hline Box 6 & 6.52 & 8.07 & 7.22 & 5.59 \\
\hline Box 7 & 10.02 & 8.07 & 7.22 & 9.25 \\
\hline Box 8 & 4.86 & 5.06 & 4.12 & 3.37 \\
\hline Box 9 & 1.07 & 1.4 & 1.03 & 1.93 \\
\hline Box 10 - right & 4.67 & 5.06 & 5.15 & 4.72 \\
\hline Refusal & 6.03 & 4.74 & 5.15 & 5.01 \\
\hline dk & 19.07 & 12.81 & 21.65 & 19.17 \\
\hline
\end{tabular}

Econometric Analysis.

Life Satisfaction

\begin{tabular}{|c|c|c|c|c|c|c|c|c|}
\hline & $\begin{array}{c}\text { Satifactio } \\
\mathrm{n}\end{array}$ & $\begin{array}{c}\text { Satifactio } \\
n\end{array}$ & Quality & Quality & Direction & Direction & $\begin{array}{l}\text { Quality } \\
\text { Better } \\
\text { Before }\end{array}$ & $\begin{array}{l}\text { Quality } \\
\text { Better } \\
\text { Before }\end{array}$ \\
\hline & $\begin{array}{c}\text { Very } \\
\text { Satisfied }\end{array}$ & $\begin{array}{c}\text { Fairly } \\
\text { Satisfied }\end{array}$ & $\begin{array}{l}\text { Very } \\
\text { Good }\end{array}$ & $\begin{array}{l}\text { Fairly } \\
\text { Good }\end{array}$ & Right & Neither & $\begin{array}{l}\text { Totally } \\
\text { Agree }\end{array}$ & $\begin{array}{l}\text { Tend to } \\
\text { Agree }\end{array}$ \\
\hline $\begin{array}{l}\text { Immediat } \\
\text { ely Before } \\
\text { the attack }\end{array}$ & 0.012 & -0.002 & & & $0.068 * * *$ & $0.043 * * *$ & & \\
\hline & 0.01 & 0 & & & 0.01 & 0.01 & & \\
\hline $\begin{array}{l}\text { Immediat } \\
\text { ely After } \\
\text { the attack }\end{array}$ & 0.021 & -0.004 & -0.005 & -0.007 & $0.077 * *$ & $0.048 * * *$ & 0.025 & 0 \\
\hline & 0.03 & 0.01 & 0.02 & 0.03 & 0.03 & 0.02 & 0.04 & 0 \\
\hline $\begin{array}{c}\text { Weeks } \\
\text { After the } \\
\text { attack }\end{array}$ & $0.032 * *$ & $-0.007 * *$ & & & $0.068 * * *$ & $0.043 * * *$ & & \\
\hline & 0.01 & 0 & & & 0.01 & 0.01 & & \\
\hline Age & 0 & 0 & 0 & 0 & $0.001 * * *$ & $0.001 * * *$ & 0.001 & 0 \\
\hline & 0 & 0 & 0 & 0 & 0 & 0 & 0 & 0 \\
\hline
\end{tabular}




\begin{tabular}{|c|c|c|c|c|c|c|c|c|}
\hline $\begin{array}{c}16-19 \\
\text { years of } \\
\text { education } \\
\end{array}$ & 0.019 & -0.001 & -0.006 & -0.013 & 0.017 & 0.012 & -0.01 & 0.001 \\
\hline & 0.02 & 0 & 0.01 & 0.03 & 0.01 & 0.01 & 0.04 & 0.01 \\
\hline $\begin{array}{c}20+\text { years } \\
\text { of } \\
\text { education }\end{array}$ & $0.049 * * *$ & $-0.007 * *$ & $0.029 *$ & 0.04 & $0.056 * * *$ & $0.034 * * *$ & $-0.170 * * *$ & -0.011 \\
\hline & 0.02 & 0 & 0.02 & 0.03 & 0.02 & 0.01 & 0.04 & 0.01 \\
\hline $\begin{array}{c}\text { Still } \\
\text { Studying } \\
\end{array}$ & $0.305 * * *$ & $-0.165 * * *$ & 0.059 & $0.060 * *$ & $0.151 * * *$ & $0.069 * * *$ & $-0.227 * * *$ & -0.039 \\
\hline & 0.05 & 0.03 & 0.05 & 0.03 & 0.04 & 0.01 & 0.06 & 0.03 \\
\hline $\begin{array}{c}\text { (Re- } \\
\text { Married/ } \\
\text { Single } \\
\text { with } \\
\text { partner } \\
\end{array}$ & $0.076 * * *$ & $-0.017 * * *$ & 0.018 & 0.033 & -0.008 & -0.004 & -0.027 & 0 \\
\hline & 0.02 & 0 & 0.01 & 0.03 & 0.01 & 0.01 & 0.03 & 0 \\
\hline $\begin{array}{c}\begin{array}{c}\text { Divorced } \\
\text { or } \\
\text { separated }\end{array} \\
\end{array}$ & $-0.041 * *$ & $-0.008^{*}$ & 0.016 & 0.03 & $-0.031 *$ & $-0.019 *$ & -0.044 & -0.001 \\
\hline & 0.02 & 0 & 0.02 & 0.03 & 0.02 & 0.01 & 0.05 & 0 \\
\hline Widowed & 0.009 & 0 & 0.026 & 0.043 & 0.001 & 0.001 & -0.013 & 0 \\
\hline & 0.02 & 0 & 0.02 & 0.03 & 0.02 & 0.01 & 0.05 & 0 \\
\hline Female & -0.012 & 0.003 & $-0.048 * * *$ & $-0.075 * * *$ & $-0.032 * * *$ & $-0.019 * * *$ & 0.024 & 0 \\
\hline & 0.01 & 0 & 0.01 & 0.02 & 0.01 & 0.01 & 0.02 & 0 \\
\hline Employed & -0.011 & 0.003 & -0.057 & $-0.052 * *$ & -0.03 & -0.015 & 0.032 & 0.005 \\
\hline & 0.04 & 0.01 & 0.04 & 0.02 & 0.03 & 0.01 & 0.06 & 0.01 \\
\hline $\begin{array}{c}\text { Not } \\
\text { working }\end{array}$ & -0.031 & 0.008 & -0.054 & $-0.047 * *$ & -0.037 & -0.019 & 0.092 & 0.007 \\
\hline & 0.04 & 0.01 & 0.04 & 0.02 & 0.03 & 0.02 & 0.06 & 0.01 \\
\hline Rural & $0.024 *$ & -0.006 & -0.008 & -0.012 & $-0.024 *$ & $-0.014 *$ & 0.032 & 0.001 \\
\hline & 0.01 & 0 & 0.01 & 0.02 & 0.01 & 0.01 & 0.03 & 0 \\
\hline $\begin{array}{c}\text { Regular } \\
\text { Trouble } \\
\text { Paying } \\
\text { Bills } \\
\end{array}$ & $-0.224 * * *$ & $0.051 * * *$ & $-0.039 * * *$ & $-0.061 * * *$ & $-0.070 * * *$ & $-0.040 * * *$ & $0.214 * * *$ & 0.004 \\
\hline & 0.02 & 0.01 & 0.01 & 0.02 & 0.02 & 0.01 & 0.03 & 0.01 \\
\hline R Adj sq. & & & & & & & & \\
\hline $\mathrm{N}$ & 3015 & 3015 & 932 & 932 & 2845 & 2845 & 932 & 932 \\
\hline
\end{tabular}

\section{Expectations}

\begin{tabular}{|c|c|c|c|c|}
\hline & Quality & quality & Confidence in future & Confidence in future \\
\hline & better & same & Totally agree & Tend to agree \\
\hline \multicolumn{5}{|l|}{ Immediately Before the attack } \\
\hline \multirow[t]{2}{*}{ Immediately After the attack } & $-0.070 * *$ & -0.01 & $-0.058 * *$ & $-0.060 * * *$ \\
\hline & 0.03 & 0.01 & 0.02 & 0.02 \\
\hline \multicolumn{5}{|l|}{ Weeks After the attack } \\
\hline \multirow[t]{2}{*}{ Age } & -0.001 & 0 & -0.001 & -0.001 \\
\hline & 0 & 0 & 0 & 0 \\
\hline 16-19 years of education & 0.018 & 0.002 & 0.003 & 0.005 \\
\hline
\end{tabular}




\begin{tabular}{|c|c|c|c|c|}
\hline & 0.03 & 0 & 0.02 & 0.02 \\
\hline \multirow[t]{2}{*}{$20+$ years of education } & -0.011 & -0.003 & $0.071 * * *$ & $0.071 * * *$ \\
\hline & 0.03 & 0.01 & 0.02 & 0.03 \\
\hline \multirow[t]{2}{*}{ Still Studying } & 0.043 & 0.002 & 0.071 & $0.071^{*}$ \\
\hline & 0.07 & 0.01 & 0.05 & 0.04 \\
\hline \multirow[t]{2}{*}{ (Re-)Married/Single with partner } & 0.002 & 0 & $0.032 *$ & $0.035^{*}$ \\
\hline & 0.03 & 0 & 0.02 & 0.02 \\
\hline \multirow[t]{2}{*}{ Divorced or separated } & 0 & 0 & 0.005 & 0.006 \\
\hline & 0.04 & 0 & 0.02 & 0.03 \\
\hline \multirow[t]{2}{*}{ Widowed } & $-0.088 * *$ & $-0.043 *$ & 0.001 & 0.001 \\
\hline & 0.04 & 0.02 & 0.03 & 0.03 \\
\hline \multirow[t]{2}{*}{ Female } & -0.003 & 0 & $-0.046^{* * *}$ & $-0.048^{* * *}$ \\
\hline & 0.02 & 0 & 0.01 & 0.01 \\
\hline \multirow[t]{2}{*}{ Employed } & -0.017 & -0.003 & -0.031 & -0.025 \\
\hline & 0.05 & 0.01 & 0.04 & 0.03 \\
\hline \multirow[t]{2}{*}{ Not working } & -0.001 & 0 & -0.042 & -0.036 \\
\hline & 0.06 & 0.01 & 0.04 & 0.03 \\
\hline \multirow[t]{2}{*}{ Rural } & 0.027 & 0.004 & -0.013 & -0.013 \\
\hline & 0.02 & 0 & 0.02 & 0.02 \\
\hline \multirow[t]{2}{*}{ Regular Trouble Paying Bills } & -0.045 & -0.007 & $-0.090 * * *$ & $-0.093 * * *$ \\
\hline & 0.03 & 0.01 & 0.02 & 0.02 \\
\hline \multicolumn{5}{|l|}{ R Adj sq. } \\
\hline $\mathrm{N}$ & 934 & 934 & 934 & 934 \\
\hline
\end{tabular}

Terrorism

\begin{tabular}{|c|c|c|}
\hline & Priority for France & Priority for oneself \\
\hline & $\mathrm{b} / \mathrm{se}$ & $\mathrm{b} / \mathrm{se}$ \\
\hline \multirow[t]{2}{*}{ Immediately After the attack } & $0.294^{* * *}$ & $0.061^{* * *}$ \\
\hline & 0.03 & 0.02 \\
\hline \multirow[t]{2}{*}{ Age } & 0.001 & 0.001 \\
\hline & 0 & 0 \\
\hline \multirow[t]{2}{*}{ 16-19 years of education } & 0.016 & 0.02 \\
\hline & 0.04 & 0.02 \\
\hline \multirow[t]{2}{*}{$20+$ years of education } & $-0.076 * *$ & -0.026 \\
\hline & 0.04 & 0.02 \\
\hline \multirow[t]{2}{*}{ Still Studying } & $-0.141^{* * *}$ & -0.003 \\
\hline & 0.05 & 0.05 \\
\hline \multirow[t]{2}{*}{ (Re-)Married/Single with partner } & -0.03 & 0.024 \\
\hline & 0.04 & 0.02 \\
\hline \multirow[t]{2}{*}{ Divorced or separated } & -0.071 & -0.01 \\
\hline & 0.05 & 0.02 \\
\hline \multirow[t]{2}{*}{ Widowed } & -0.079 & 0.004 \\
\hline & 0.05 & 0.02 \\
\hline \multirow[t]{2}{*}{ Female } & $0.042^{*}$ & -0.011 \\
\hline & 0.02 & 0.01 \\
\hline \multirow[t]{2}{*}{ Employed } & -0.04 & 0.003 \\
\hline & 0.07 & 0.04 \\
\hline Not working & -0.031 & -0.007 \\
\hline
\end{tabular}




\begin{tabular}{|l|r|r|}
\hline & 0.07 & 0.04 \\
\hline Rural & -0.038 & -0.03 \\
\hline & 0.03 & 0.02 \\
\hline Regular Trouble Paying Bills & -0.035 & -0.023 \\
\hline & 0.03 & 0.02 \\
\hline R Adj sq. & & 1000 \\
\hline N & 1000 & 1 \\
\hline
\end{tabular}

Trust and Political Orientation

\begin{tabular}{|c|c|c|c|c|}
\hline & Political Parties & Local Gov. & National Gov. & $\begin{array}{c}\text { Political } \\
\text { Orientation }\end{array}$ \\
\hline & Tend to Trust & Tend to Trust & Tend to Trust & \\
\hline \multirow[t]{2}{*}{ Immediately Before the attack } & & & & $-0.191 *$ \\
\hline & & & & 0.11 \\
\hline \multirow[t]{2}{*}{ Immediately After the attack } & 0.041 & 0.043 & $0.133^{* * *}$ & -0.145 \\
\hline & 0.03 & 0.06 & 0.04 & 0.27 \\
\hline \multirow[t]{2}{*}{ Weeks After the attack } & & & & -0.041 \\
\hline & & & & 0.11 \\
\hline \multirow[t]{2}{*}{ Age } & 0 & 0.001 & 0.001 & -0.002 \\
\hline & 0 & 0 & 0 & 0 \\
\hline \multirow[t]{2}{*}{$16-19$ years of education } & -0.041 & -0.048 & -0.044 & 0.118 \\
\hline & 0.03 & 0.05 & 0.04 & 0.14 \\
\hline \multirow[t]{2}{*}{$20+$ years of education } & 0.001 & 0.026 & 0.018 & $-0.467^{* * *}$ \\
\hline & 0.03 & 0.06 & 0.05 & 0.15 \\
\hline \multirow[t]{2}{*}{ Still Studying } & 0.035 & $0.205 * *$ & 0.109 & -0.494 \\
\hline & 0.08 & 0.1 & 0.1 & 0.3 \\
\hline \multirow[t]{2}{*}{ (Re-)Married/Single with partner } & -0.013 & 0.026 & 0.027 & 0.193 \\
\hline & 0.03 & 0.05 & 0.04 & 0.13 \\
\hline \multirow[t]{2}{*}{ Divorced or separated } & $-0.080 * * *$ & -0.061 & -0.036 & 0.132 \\
\hline & 0.03 & 0.06 & 0.05 & 0.18 \\
\hline \multirow[t]{2}{*}{ Widowed } & -0.002 & 0.001 & -0.014 & $0.531^{* *}$ \\
\hline & 0.04 & 0.07 & 0.06 & 0.21 \\
\hline \multirow[t]{2}{*}{ Female } & 0.011 & -0.053 & -0.016 & $-0.284 * * *$ \\
\hline & 0.02 & 0.03 & 0.03 & 0.09 \\
\hline \multirow[t]{2}{*}{ Employed } & 0 & 0.09 & 0.059 & -0.406 \\
\hline & . & 0.09 & 0.07 & 0.27 \\
\hline
\end{tabular}




\begin{tabular}{|c|c|c|c|c|}
\hline Not working & 0 & 0.026 & 0.016 & -0.343 \\
\hline &. & 0.09 & 0.07 & 0.27 \\
\hline Rural & -0.006 & 0.003 & $-0.066^{*}$ & -0.102 \\
\hline Regular Trouble Paying Bills & 0.02 & 0.04 & 0.04 & 0.12 \\
\hline & 0.005 & -0.075 & $-0.116^{* * *}$ & -0.036 \\
\hline R Adj sq. & 0.03 & 0.05 & 0.04 & 0.14 \\
\hline $\mathrm{N}$ & & & & \\
\hline
\end{tabular}

